ISSN 0103-9954

\title{
AVALIAÇÃO DE CUSTOS DE DOIS MODELOS DE HARVESTER NO CORTE DE EUCALIPTO
}

\author{
EVALUATION OF COSTS OF TWO HARVESTER MODELS IN THE CUT OF Eucalyptus
}

\author{
Elizabeth Neire da Silva ${ }^{1}$ Carlos Cardoso Machado ${ }^{2}$ Nilton Cesar Fiedler ${ }^{3}$ Haroldo Carlos Fernandes ${ }^{4}$ \\ Marcos Oliveira de Paula ${ }^{5}$ Flávio Cipriano do Carmo $^{6}$ Gisele Rodrigues Moreira $^{7}$ \\ Fernando Eugênio Coelho ${ }^{8}$
}

\begin{abstract}
RESUMO
O presente estudo teve o objetivo de realizar a avaliação de custos de dois modelos de harvester, na colheita de madeira de eucalipto. A pesquisa avaliou a derrubada e o processamento da árvore, com idade de 6 anos, no sistema de toras curtas, para dois modelos de harvester, em áreas localizadas nos municípios de Conceição da Barra - ES e Caravelas - BA. Avaliaram-se dois modelos de harvester, PC-228 SHO e PC-200 LC, da marca Komatsu. As informações foram coletadas durante um período de 6 meses, que compreende os meses de março a agosto de 2010, pelo método de censo. Calcularam-se os custos operacionais dos dois modelos de harvester, pelo método contábil, o qual utiliza valores estimados em reais. Realizou-se ainda a análise de sensibilidade de custos para os elementos que mais contribuem com o custo final. Obteve-se o custo operacional para os modelos PC 200 e PC 228 de R\$156,95 e R\$168,84 por hora efetiva, respectivamente. Em relação aos custos operacionais totais, os custos mais significativos foram: combustível, manutenção e reparos e depreciação, com $24,41 \%, 22,39 \%$ e $19,08 \%$, respectivamente. Na análise de sensibilidade, simulando uma situação em que a empresa consiga uma economia real de $10 \%$ em cada um desses itens, a mesma poderá obter uma redução no custo de produção em $7 \%$, para as duas máquinas avaliadas.

Palavras-chave: harvester; colheita mecanizada; tempos e produtividade florestal.
\end{abstract}

\section{ABSTRACT}

The present study aimed to carry through the evaluation of costs of two models of harvester in the wood harvesting of Eucalyptus wood. The research evaluated the 6-year old tree fallen and processing, in the cut-to-length system for two models of harvester, in areas located in the cities of Conceição da Barra,

1 Engenheira Florestal, Dr., Professora Adjunta do Departamento de Ciências Florestais e da Madeira, Universidade Federal do Espírito Santo, Av. Governador Lindemberg, 316, Bairro Centro, CEP 29550-000, Jerônimo Monteiro (ES), Brasil. elizabeth@cca.ufes.br

2 Engenheiro Florestal, Dr., Professor Titular do Departamento de Engenharia Florestal, Universidade Federal de Viçosa, Av. P.H. Rolfs, s/n, Campus Universitário, CEP 36570-000, Viçosa (MG), Brasil. machado@ufv.br

3 Engenheiro Florestal, Dr., Professor Adjunto do Departamento de Ciências Florestais e da Madeira, Universidade Federal do Espírito Santo, Av. Governador Lindemberg, 316, Bairro Centro, CEP 29550-000, Jerônimo Monteiro (ES), Brasil. fiedler.pq@cnpq.br

4 Engenheiro Florestal, Dr., Professor Titular do Departamento de Engenharia Florestal, Universidade Federal de Viçosa, Av. P.H. Rolfs, s/n, Campus Universitário, CEP 36570-000, Viçosa (MG), Brasil. machado@ufv.br

5 Engenheiro Civil, Dr., Professor Adjunto do Departamento de Engenharia Rural, Universidade Federal do Espírito Santo, Alto Universitário s/n, Guararema, CEP 29500-000, Alegre (ES), Brasil. marcos.paula@ufes.br

6 Engenheiro Florestal, Msc., Doutorando em Ciências Florestais, Programa de Pós-Graduação em Ciências Florestais, Universidade Federal do Espírito Santo, Av. Governador Lindemberg, 316, Bairro Centro, CEP 29550000, Jerônimo Monteiro (ES), Brasil. flaviocipriano@hotmail.com

7 Engenheira Agrônoma, Dra ${ }^{\mathrm{a}}$, Professora Adjunta do Departamento de Engenharia Rural, Universidade Federal do Espírito Santo, Alto Universitário s/n, Guararema, CEP 29500-000, Alegre (ES), Brasil. gisele.moreira@ufes.br

8 Engenheiro Florestal, Mestrando em Ciências Florestais, Programa de Pós-Graduação em Ciências Florestais, Universidade Federal do Espírito Santo, Av. Governador Lindemberg, 316, Bairro Centro, CEP 29550-000, Jerônimo Monteiro (ES), Brasil. coelho.fernando@yahoo.com.br

Recebido para publicação em 17/10/2011 e aceito em 25/03/2013

Ci. Fl., v. 24, n. 3, jul.-set., 2014 
Espírito Santo state and Caravelas, Bahia state. It was evaluated the models of harvester PC-228 SHO and PC-200 LC, of the Komatsu Mark. The information was collected during a period of 6 months, from March to August 2010, by the method of census. It was calculated the operational costs of the two models of harvester, for the countable method, which uses values in 'Reais', the Brazilian currency. It was conducted further analysis of cost sensitivity for the elements that contribute most to the final cost. We obtained the operating cost models for PC 200 and PC 228 of R\$156,95 and R\$168,84 per effective hour, respectively. In relation with the total operational costs, the most significant costs were: fuel, maintenance, and repairs/ depreciation, with $24.41 \%, 22.39 \%$ and $19.08 \%$, respectively. In the sensitivity analysis, simulating a situation where the company gets a real economy of $10 \%$ in each one of these items, the same one will be able to get a reduction in the cost of production in $7 \%$, for the two evaluated machines.

Keywords: harvester; mechanized harvesting; forest productivity and time.

\section{INTRODUÇÃO}

A crescente demanda mundial por madeira, juntamente com os aspectos edafoclimáticos favoráveis contribuem para que o Brasil tenha papel de destaque no cenário mundial, o que proporciona ao País poder de influência nas decisões relacionadas ao setor florestal.

Economicamente, o setor tem sido responsável, anualmente, na formação econômica do País, por aproximadamente $4 \%$ do Produto Interno Bruto (PIB). A estimativa total de empregos (primário e processamento industrial) no segmento de florestas plantadas, em 2009, foi de 3,9 milhões incluindo os diretos (535 mil), indiretos (1,26 milhão) e empregos resultantes do efeito renda (2,16 milhões), gerando US\$ 5,6 bilhões em divisas de exportações em 2009 (ABRAF, 2010).

Dentro do cenário de expansão da produção e a busca por competitividade, o Brasil é avaliado por experientes profissionais como um dos países que possui as melhores condições de atrair investimentos para implantação de empreendimentos florestais (SILVA, 2008).

Machado e Lopes (2000) afirmam que a colheita e o transporte florestal são responsáveis por mais da metade do custo final da madeira colocada no centro consumidor. Por esse motivo, a seleção de máquinas e equipamentos e o desenvolvimento de sistemas operacionais constituem o grande desafio para a redução dos custos operacionais de colheita e transporte florestal.

Com o aumento da demanda de madeira para atender à produção de celulose e fabricação de painéis no Brasil, a evolução da mecanização da colheita florestal intensificou-se, desencadeando um processo contínuo de avaliação dos rendimentos operacionais e dos custos, devido à colheita resultar em um percentual de custo elevado (SIMÕES, 2008).

O custo operacional de uma máquina, segundo Harry et al. (1991), é o somatório de todos os custos resultantes de sua aquisição e operação. O seu conhecimento é uma etapa de fundamental importância para o planejamento e o controle de sua utilização. A variação deste custo é influenciada, principalmente, pela eficiência operacional e pela jornada de trabalho.

A utilização de equipamentos de alta tecnologia, como o harvester, aumentou o rendimento operacional, a segurança no trabalho e colocou os custos entre os mais competitivos do mundo (MAGALHÃES e KATZ, 2010).

A redução dos custos da colheita é, segundo Rezende et al. (1997), vital para qualquer empresa, uma análise detalhada e por partes dos custos nos diferentes métodos de colheita tem um papel importante no entendimento dos mesmos, além de facilitar os estudos com o objetivo de reduzi-los.

Desta forma, o presente estudo visa avaliar os aspectos custos do corte mecanizado de madeira de eucalipto com harvester dos dois modelos de harvesters, visando contribuir para a diminuição dos custos operacionais e de produção.

\section{MATERIAL E MÉTODOS}

\section{Área de estudo}

A área de estudo situa-se na Região Sudeste do Brasil, nos municípios Conceição da Barra - ES e Caravelas - BA. O relevo é caracterizado como praticamente plano a suave ondulado com declividade máxima de até $5 \%$, altitudes entre 10 e 50 m, clima tropical úmido, precipitação média anual de $1350 \mathrm{~mm}$, sendo o período chuvoso de 
outubro a dezembro e o período seco de julho a setembro (INMET, 2011).

Avaliaram-se os custos para o trabalho em sistema de colheita de toras curtas com operações mecanizadas de abate e processamento. Na operação de corte foram utilizados dois modelos diferentes de harvesters, sendo a extração realizada por forwarder. A operação é realizada em dois turnos diários de 8 horas.

Nesta pesquisa avaliaram-se dois modelos de harvester, PC-228 SHO e PC-200 LC, fabricado pela Komatsu, com configuração florestal, sobre esteiras. Os dois modelos avaliados operam com um cabeçote harvester Valmet $370 \mathrm{E}$, com peso de $1.600 \mathrm{~kg}, 6$ facas, com vida útil estimada de 15.000 horas, sem valor residual.

\section{Método de amostragem}

As informações referentes às características avaliadas do corte florestal mecanizado foram coletadas durante um período de 6 meses, que compreende os meses de março a agosto de 2010. A coleta de dados foi realizada com uso de um estudo de tempo e movimentos, caracterizado pelo método de tempos contínuos com uso de cronômetro digital e formulário para registro dos dados. $\mathrm{O}$ acompanhamento das operações e fases foi in loco, no andamento normal das atividades na empresa, sem qualquer interferência. O estudo de tempos foi realizado para obter o rendimento das atividades e posterior cálculo dos custos de produtividade (ZINKEVIČIUS et al., 2012).

\section{Determinação dos custos}

$\mathrm{Na}$ determinação dos custos operacionais dos dois diferentes modelos de harvester utilizou-se o método contábil, o qual utiliza valores estimados e reais. Os custos foram estimados pela metodologia proposta pela FAO segundo Machado e Malinovski (1988) e Mederski (2006).

Nesta metodologia os custos são classificados em componentes de custo, em que os custos da máquina são compostos por:

- Custos de Maquinário (Custos fixos e custos variáveis);

- Custos de pessoal (mão de obra);

- Custos de administração.

Assim, o custo final obtido foi o somatório dos custos de maquinário (fixos e variáveis), pessoal (mão de obra) e de administração em horas efetivas, obtidas pela Equação 1:

$$
C T=(C F+C V)+C A D+C M O
$$

Em que: $\mathrm{CT}=$ Custos totais; $\mathrm{CF}=$ Custos fixos; $\mathrm{CV}=$ Custos variáveis; $\mathrm{CAD}=$ Custos de administração; $\mathrm{CMO}=$ Custos com mão de obra.

\section{Custo de maquinário}

\section{Custos fixos}

Juros

Os juros $\left(\mathrm{J}=\mathrm{R} \$ \mathrm{hf}^{-1}\right)$ foram calculados pela aplicação de uma taxa de juros ao investimento, correspondente ao capital proporcionado por agência financeira, aqui obtida conforme Equações 2 e 3.

$$
J=\frac{(I M A \times i)}{H f}
$$

Em que: $\mathrm{J}=$ juros $\left(\mathrm{R} \$ . \mathrm{Hf}^{-1}\right) ; \mathrm{i}=$ taxa anual de juros simples (\%); Hf = horas efetivas de uso anual; IMA $=$ Investimento médio anual.

$$
I M A=\frac{V a[(t+1)+V r(t-1)]}{(2 \times t)}
$$

Em que: $V a=$ valor de aquisição da máquina $(\mathrm{R} \$) ; \mathrm{Vr}=$ valor residual da máquina $(\mathrm{R} \$) ; \mathrm{t}=$ vida útil em anos.

\section{Seguros}

Os seguros são feitos contra riscos de incêndios e acidentes. $\mathrm{O}$ valor anual é estabelecido entre 5 a $10 \%$ do valor de aquisição da maquina ou equipamento. Neste caso, adotou-se a taxa de $5 \%$, aplicando a Equação 4.

$$
S=\frac{(V a \times P)}{H f}
$$

Em que: $\mathrm{S}=$ seguros $\left(\mathrm{R} \$ \cdot \mathrm{Hf}^{-1}\right) ; \mathrm{Va}=$ valor de aquisição da máquina; $\mathrm{P}=$ percentagem expressa em forma decimal; $\mathrm{Hf}=$ horas efetivas de uso anual.

Impostos

Correspondem a valores anuais relacionados com a propriedade da máquina ou equipamento (IPI, 
ICMS e frete), calculados conforme Equação 5.

$$
I=\frac{I a}{H f}
$$

Em que: $\mathrm{Ia}=$ Impostos anuais $(\mathrm{R} \$) ; \mathrm{Hf}=$ horas efetivas de uso anual.

\section{Depreciação}

A depreciação é um modo de recuperar o investimento original de uma máquina. Aqui, utiliza-se a depreciação linear, conforme se observa na Equação 6:

$$
D=\frac{(V a-V r)}{H}
$$

Em que: $\mathrm{D}=$ Depreciação; Va $=$ Valor de aquisição da máquina $(\mathrm{R} \$) ; \mathrm{Vr}=$ valor residual $(\mathrm{R} \$)$; $\mathrm{H}=$ vida econômica da máquina (horas totais).

\section{Custos variáveis}

São os custos que variam, proporcionalmente, com a quantidade produzida ou com o uso da máquina, tais como os custos de combustível, lubrificantes, óleo hidráulico, pneus, remuneração de pessoal e manutenção e reparos.

\section{Combustivel}

O consumo de combustível é função da potência do motor, fator de carga, altitude, temperatura, tipo de combustível, etc. Foi calculado multiplicando-se o consumo médio horário de cada máquina, segundo sua média semestral, pelo preço atual de mercado do óleo diesel (Equação 7).

$$
C C=C b(\text { mensurada }) \times p
$$

Em que: $\mathrm{Cb}=$ Consumo de combustível por hora efetiva de trabalho $\left(\mathrm{L} \mathrm{He}^{-1}\right) ; \mathrm{P}=$ preço atual de mercado (R\$).

\section{Manutenção e reparos}

Consistem basicamente em mão de obra e encargos sociais, além de peças de reposição e outros materiais. Neste caso foram obtidos segundo a Equação 8:

$$
M R=\frac{V a}{H}
$$

Em que: $\mathrm{MR}=$ manutenção e reparos; $\mathrm{Va}=$ valor de aquisição; $\mathrm{H}=$ vida econômica da máquina (horas totais).

\section{Custos da mão de obra}

São custos variáveis, sendo formados pelos custos diretos e indiretos com o operador da máquina, obtidos pela Equação 9.

$$
C M O=\frac{12 \times \operatorname{Sm}(1+s)}{H f}
$$

Em que: $\mathrm{Sm}=$ Salário mensal; $\mathrm{s}=$ fator de encargos sociais; $\mathrm{Hf}=$ horas efetivas de uso anual.

Para efeito de cálculo adotou-se o mesmo fator de encargos sociais utilizados por Burla (2008), $\log$ o $s=1,2$, que significa a incidência de $120 \%$ sobre o valor do salário do funcionário.

\section{Custo de Administração}

São os custos indiretos, relacionados com a administração do trabalho e maquinário. Neste caso adotou-se k=10\%, segundo a Equação 10 .

$$
C A D=C D \times K
$$

Em que: $\mathrm{CD}=$ custos diretos do maquinário e mão de obra; $\mathrm{K}=$ coeficiente de administração que varia entre 10 a $20 \%(0,10 \leq k \geq 0,20)$.

Em todos os cálculos, a conversão dos valores em reais para dólar foi feita baseando-se na média anual de 2010, segundo a Fundação Getúlio Vargas (FGV, 2011).

\section{RESULTADOS E DISCUSSÕES}

\section{Custos operacionais}

$\mathrm{Na}$ situação real local, na qual ambos os modelos de harvester trabalham por um período de 16 horas diárias, e o fato de que a presença de alguns custos agregados apenas no período diurno de trabalho interferiria na avaliação econômica do período noturno, procederam-se os cálculos referentes aos custos operacionais apenas para a jornada de trabalho completa, com o intuito de 
TABELA 1:Valores utilizados no cálculo do custo operacional das máquinas avaliadas.

TABLE 1: Values used in the calculation of the operational cost of the evaluated machines.

\begin{tabular}{lcc}
\hline \multicolumn{1}{c}{ Itens } & PC 200 & PC 228 \\
\hline Taxa de juros anual (\%) & 11,322 & 11,322 \\
Valor de aquisição (R\$) & $878.800,00$ & $928.800,00$ \\
Vida útil (horas) & 25.000 & 25000 \\
Valor residual (R\$) & $130.000,00$ & $140.000,00$ \\
Taxa de conversão do dólar & 1,76 & 1,76 \\
Consumo médio diesel por hora (1/h) & 19,98 & 19,75 \\
Consumo médio de óleo hidráulico $(1 / \mathrm{h})$ & 0,21 & 0,62 \\
Consumo médio de óleo motor $(1 / \mathrm{h})$ & 0,13 & 0,12 \\
Custo do diesel (R\$) & 1,92 & 1,92 \\
Custo óleo hidráulico (R\$) & 17,80 & 17,80 \\
Custo de óleo motor (R\$) & 10,80 & 10,80 \\
Produtividade horária (m & 18,57 & 19,88 \\
Horas trabalhadas mensais (média) & 385,21 & 402,04 \\
\hline
\end{tabular}

representar o custo real dos harvester.

Adotou-se um valor residual das máquinas em $20 \%$ do valor de aquisição da máquina base (sem o cabeçote), com taxa de juros baseada no valor médio anual do IGP-M para 2010, e o valor de conversão do dólar obtido pela média anual de 2010 (FGV, 2011). Os valores utilizados no cálculo do custo dos dois diferentes modelos de harvester podem ser observados na tabela 1 .

Aplicando-se a metodologia da FAO, segundo Machado e Malinovski (1988), obteve-se o custo operacional para os modelos PC 200 e PC 228 de R\$ 156,95 e R $\$ 168,84$, respectivamente, conforme se observa na Tabela 2.

A distribuição dos custos em ambos os harvesters foi semelhante. Os custos fixos

TABELA 2: Componentes do custo operacional dos harvesters.

TABLE 2: Components of the operational cost of harvesters.

\begin{tabular}{lcc}
\hline \multirow{2}{*}{ Componentes de custo } & \multicolumn{2}{c}{ Custo unitário (R\$) } \\
\cline { 2 - 3 } & PC 200 & PC 228 \\
\hline Depreciação & 29,95 & 31,55 \\
Seguros & 1,76 & 1,86 \\
Juros & 2,53 & 2,67 \\
Impostos & 5,99 & 6,31 \\
Soma - Custos Fixos & 40,23 & 42,39 \\
Combustível & 38,36 & 37,92 \\
Óleo hidráulico & 3,79 & 10,98 \\
Óleo motor & 1,40 & 1,29 \\
Manutenção e reparos & 35,15 & 37,15 \\
Soma - Custos Variáveis & 78,70 & 87,35 \\
Operadores & 23,75 & 23,75 \\
Soma - Custos de Mão de obra & 23,75 & 23,75 \\
Custos de Administração & 14,27 & 15,35 \\
Custo Total (R h $^{-1}$ ) & 156,95 & 168,84 \\
Custo de produção $\left(\mathrm{R} \$\right.$ m $\left.^{-3}\right)$ & 8,45 & 8,49 \\
\hline
\end{tabular}


corresponderam a $25,63 \%$ e $25,11 \%$ para a $\mathrm{PC}$ 200 e PC 228, respectivamente. O componente mais relevante dos custos foram os custos variáveis, ou seja, custos de combustíveis, óleos e manutenção das máquinas. A distribuição dos elementos que compõem o custo operacional das máquinas é ilustrada na Figura 1.

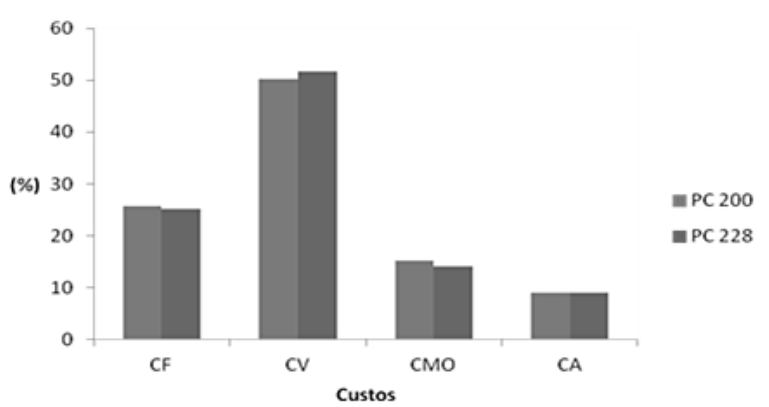

FIGURA 1: Distribuição dos custos fixos (CF), custos variáveis (CV), custos com mão de obra (CMO) e custos de administração (CA), em \%, dos harvesters.

FIGURE 1: Distribution of fixed costs (FC), variable costs $(\mathrm{VC})$, costs of labor work $(\mathrm{CMO})$ and administration costs (CA), in $\%$ of harvesters.

A maior parte dos custos fixos representa o custo de depreciação da máquina que, em ambos os casos, representou $74,4 \%$ dos custos fixos.

Os itens mais significativos nos custos variáveis foram o combustível e a manutenção e reparos. Juntos eles são responsáveis por mais de $50 \%$ dos custos variáveis totais.

De acordo com Fernandes e Leite (2001), o consumo de combustível corresponde a um dos principais itens formadores do custo operacional da máquina, bem como, constitui um indicativo da eficiência do processo de conversão de energia do sistema mecanizado utilizado na operação de colheita, fato observado também neste estudo, onde o custo de combustível representa $24,41 \%$ dos custos operacionais.

Em relação aos custos operacionais totais, os custos mais significativos foram: combustível, manutenção e reparos e depreciação, com $24,41 \%$, $22,39 \%$ e $19,08 \%$, respectivamente.

Os custos operacionais encontrados foram superiores aos encontrados por Simões (2008), para o modelo 200 LC John Deere, que foi de US\$
78,78 por hora efetiva de trabalho e por Silva (2008), para o modelo Caterpillar 320 CC. No entanto, ambas as máquinas utilizadas nesta comparação apresentaram esse valor médio para uma área com volume individual de árvores superiores aos aqui encontrados.

A mesma situação ocorre quando se compara os resultados com os de Burla (2008), que, por meio de modelagem de dados, obteve um custo de US\$ 4,64 (PC 200= US\$ 4,80; PC 228=US\$ 4,83), para uma floresta com volume individual de $0,21 \mathrm{~m}^{3}$, em $0 \%$ de declividade, para o modelo 1270 John Deere.

Os custos de produção encontrados foram obtidos considerando-se a produtividade média $\left(\mathrm{m}^{3} /\right.$ hora) no período avaliado de cada máquina, que foi de $18,57 \mathrm{~m}^{3} / \mathrm{h}$ (PC 200) e $19,88 \mathrm{~m}^{3} / \mathrm{h}$ (PC 228), o que representa um valor de $\mathrm{R} \$ 8,45$ (US\$ 4,80) e R \$ 8,49 (US\$ 4,83) por $\mathrm{m}^{3}$, respectivamente.

Considerando-se a situação real em que o estudo foi realizado, a indústria possui uma demanda mensal de $50.000 \mathrm{~m}^{3}$ de madeira. Apesar de aparentemente representar um valor pouco significativo, a diferença unitária por metro cúbico produzido representa uma economia da PC $200 \mathrm{em}$ relação a PC 228 de 4 centavos de real, representando uma economia real de aproximadamente $\mathrm{R} \$$ 2.050,00 mensais e R\$ 25.000 anuais, o que representaria a cobertura dos custos anuais de um adicional de $2.900 \mathrm{~m}^{3}$ de madeira.

\section{Análise de sensibilidade}

A análise de sensibilidade tem como objetivo determinar o grau de variação dos resultados e dos indicadores de viabilidade diante de alterações nas variáveis mais relevantes de um determinado projeto (OLIVEIRA, 2009).

Para as máquinas avaliadas, as variáveis que mais influenciaram os custos de produção, em percentuais, foram: manutenção e reparos, combustível e depreciação. Desta forma, utilizandose uma variação em $20 \%$ (10\% para mais e para menos) dos custos mais relevantes, observa-se como esses fatores influenciam na diferença do custo de produção entre as duas máquinas.

\section{Combustível}

Uma alteração no custo de combustível interfere na diferença do custo final de produção entre as duas máquinas. Observa-se na Figura 2, a existência de uma tendência de diminuição 
na diferença de custos entre as duas máquinas, conforme o custo do combustível aumenta. Com base nos resultados obtidos observou-se que essa tendência diminui em $\mathrm{R} \$ 0,02$ a cada $1 \%$ de aumento do custo do combustível.

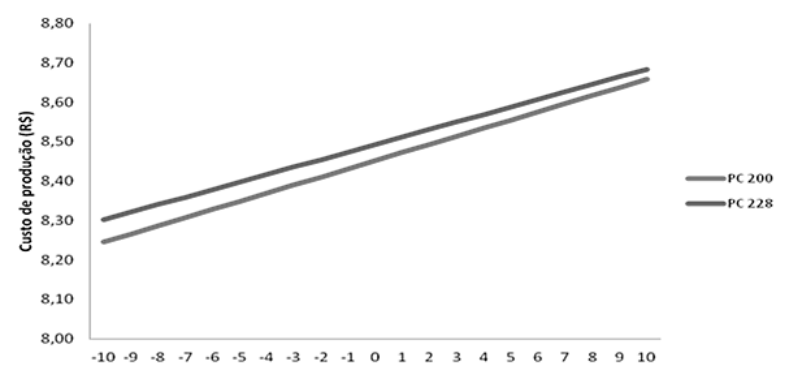

FIGURA 2: Comparação dos custos de produção com a variação da depreciação em $20 \%$ do valor original.

FIGURE 2: Comparison of production costs with the change in depreciation at $20 \%$ of original value.

\section{Manutenção e reparos}

Diferentemente do custo de combustível, uma alteração no custo de manutenção e reparos não interfere na diferença do custo final de produção entre as duas máquinas. Observa-se na figura 1, que a diferença de custos das duas máquinas mantém-se conforme o custo de manutenção e reparos aumenta ou diminui. Com base nos resultados obtidos observou-se que houve uma diminuição em R\$ 0,02 a cada $1 \%$ de aumento do custo de manutenção e reparos, que ocorreu na mesma proporção do custo de combustível.

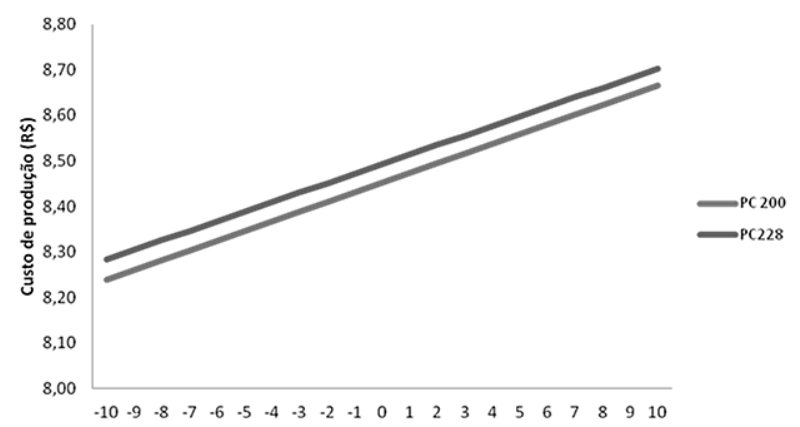

FIGURA 3: Comparação dos custos de produção com a variação do custo de manutenção e reparos em $20 \%$ do valor original.

FIGURE 3: Comparison of production costs to changes in the cost of maintenance and repairs at $20 \%$ of the original value.

\section{Depreciação}

Variando a depreciação da máquina em $20 \%$ do valor inicialmente obtido, observa-se que, em ambas as situações (PC 200 e PC 228), o custo de produção aumenta na mesma proporção para os dois modelos (Figura 4). Cada 1\% de aumento no custo da depreciação resulta de $\mathrm{R} \$ 0,02$ à $\mathrm{R} \$ 0,03$ no custo final de produção da madeira $\left(\mathrm{m}^{3}\right)$ de ambas as máquinas, enquanto a diferença no custo entre elas se mantém em R \$ 0,04.

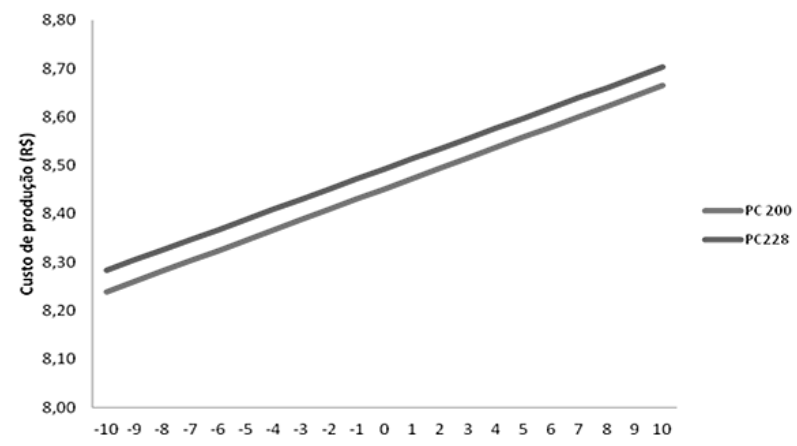

FIGURA 4: Comparação dos custos de produção com a variação da depreciação em $20 \%$ do valor original.

FIGURE 4: Comparison of production costs with the change of depreciation at $20 \%$ of original value.

\section{Análise integrada}

Com base nos resultados obtidos para os componentes de custo: combustível, manutenção e reparos e depreciação, simulando uma situação em que a empresa consiga uma economia real de $10 \%$ em cada um desses itens, a mesma poderá obter uma redução no custo de produção em $7 \%$, para as duas máquinas avaliadas, o que representa uma economia real de $\mathrm{R} \$ 0,627$ por $\mathrm{m}^{3}$ produzido para a PC 200 e $\mathrm{R} \$ 0,606$ por $\mathrm{m}^{3}$ produzido para a PC 228 (Tabela 3).

\section{CONCLUSÕES}

O menor custo operacional e de produção foi observado no modelo PC 200;

Os itens que mais influenciaram nos custos finais de ambas as máquinas foram: combustível, manutenção e reparos e depreciação, respectivamente;

A otimização das atividades que compõem 
TABELA 3: Acumulação de redução de custos obtida com a diminuição em $10 \%$ dos componentes de custo mais relevantes.

TABLE 3: Accumulation of cost reduction obtained with the decrease in $10 \%$ of the most significant cost components.

\begin{tabular}{ccccccc}
\hline Máquina & $\mathrm{CC}(\mathrm{R} \$)$ & $\mathrm{MR}(\mathrm{R} \$)$ & $\mathrm{Dp}(\mathrm{R} \$)$ & Total & $\mathrm{CP}(\mathrm{R} \$)$ & Diferença* $(\%)$ \\
\hline PC 200 & 0,207 & 0,208 & 0,213 & 0,627 & 7,823 & $-7,43$ \\
PC 228 & 0,191 & 0,206 & 0,209 & 0,606 & 7,884 & $-7,17$ \\
\hline
\end{tabular}

Em que: * Refere-se à subtração do custo obtido pelo custo obtido com a análise de sensibilidade. $\mathrm{CC}=$ Custo de combustível; $\mathrm{MR}=$ Custo de manutenção e reparos; $\mathrm{Dp}=$ Depreciação; $\mathrm{CP}=$ Custo de produção.

os custos operacionais, especialmente aquelas que mais influem nesses custos, resultam em uma economia de aproximadamente $7 \%$ no custo final por $\mathrm{m}^{3}$ produzido, em ambas as máquinas.

\section{AGRADECIMENTOS}

Universidade Federal de Viçosa (UFV); Universidade Federal do Espírito Santo (UFES); Conselho Nacional de Pesquisa e Desenvolvimento (CNPq); Fundação de Amparo à Pesquisa do Espírito Santo (Fapes); Fibria S/A.

\section{REFERÊNCIAS BIBLIOGRÁFICAS}

ABRAF. Anuário Estatístico: ano base 2009/ ABRAF. - Brasília, 2010. 136 p.

BURLA, E. R. Avaliação técnica e econômica do harvester na colheita e processamento de madeira em diferentes condições de declividade e produtividade florestal. 2008. 70 f. Dissertação (Mestrado em Engenharia Agrícola). UFV, Viçosa, 2008.

Fundação Getúlio Vargas. FGV. Disponível em: http://portalibre.fgv.br/main.jsp. Acesso em 14 de janeiro de 2011.

FERNANDES, H. C.; LEITE, A. M. P. Proposta de uma metodologia para ensaio de máquinas colhedoras de madeira. In: SIMPÓSIO BRASILEIRO SOBRE COLHEITA E TRANSPORTE FLORESTAL, 5., 2001, Porto Seguro. Anais... Viçosa: SIF/UFV, 2001. p. 227- 239.

HARRY G. G. et al. Análise dos efeitos da eficiência no custo operacional de máquinas florestais. In: SIMPÓSIO BRASILEIRO SOBRE EXPLORAÇÃO E TRANSPORTE FLORESTAL, 1., 1991, Belo Horizonte. Anais... Belo Horizonte: UFV/SIF, 1991.

MACHADO C. C.; LOPES E. S. Análise da influência do comprimento de toras de eucalipto na produtividade e custo da colheita e transporte florestal. Cerne, v. 6, n. 2, p. 124-129, 2000.

MACHADO, C. C.; MALINOVSKI, J. R. Ciência do trabalho florestal. Viçosa: Universidade Federal de Viçosa, 1988. 65 p.

MAGALHÃES, P. A. D; KATZ, I. Estudo da viabilidade econômica da mecanização do processo de colheita florestal com harvester em uma indústria madeireira. Tékhne Lógos, Botucatu, v. 2, n. 1, p.72 a 91, out. 2010.

MEDERSKI, P. S. A comparison of harvesting productivity and costs in thinning operations with and without midfield. Forest Ecology and Management. v. 224, p. 286-296, 2006

REZENDE, J.L. et al. Análise técnica e de custos de métodos de colheita e transporte florestal. Lavras: UFLA, 1997. 50 p. (Boletim Agropecuário, 22)

SILVA, E. N. Avaliação técnica e econômica do corte de pinus com harvester. 2008. 60 f. Dissertação (Mestrado em Ciência Florestal) Universidade Federal de Viçosa, Viçosa, 2008.

SIMÕES, D. Avaliação econômica de dois sistemas de colheita florestal mecanizada de eucalipto. 2008. 105 f. Dissertação (Mestrado em Agronomia / Energia na Agricultura) - Faculdade de Ciências Agronômicas / Universidade Estadual Paulista, Botucatu, 2008.

OLIVEIRA, R.J. Avaliação econômica da utilização de cabos aéreos na colheita florestal em regiões montanhosas. 2009. Dissertação (Mestrado em Ciência Florestal) - Universidade Federal de Viçosa, 2008.

ZINKEVIČIUUS R. et al. Comparison of harvester and motor-manual logging in intermediate cuttings of deciduous stands. Turkish Journal Of Agriculture \& Forestry [serial online]. October 2012. 